EUROPEAN ORGANIZATION FOR NUCLEAR RESEARCH

European Laboratory for Particle Physics

Large Hadron Collider Project

LHC Project Report 494

\title{
MAGNETIC FIELD CALCULATIONS INCLUDING THE IMPACT OF PERSISTENT CURRENTS IN SUPERCONDUCTING FILAMENTS
}

\author{
M. Aleksa, S. Russenschuck, C. Völlinger
}

\begin{abstract}
The magnetic field in the coils of superconducting magnets induces so-called persistent currents in the filaments. Persistent currents are bipolar screening currents that do not decay due to the lack of resistivity. The NbTi-filaments are type II superconductors and can be described by the critical state model. This paper presents an analytical hysteresis model of the filament magnetization due to persistent currents which takes into account the changing magnetic induction inside the filament. This model is combined with numerical field computation methods, taking local saturation effects in the ferromagnetic yoke into consideration.
\end{abstract}

LHC Division

Presented at the 13th Conference on the Computation of Electromagnetic Fields (COMPUMAG)

2-5 June 2001, Lyon-Evian, France

\footnotetext{
Administrative Secretariat

LHC Division

CERN

CH - 1211 Geneva 23

Switzerland

Geneva, 27 August 2001
} 


\title{
Magnetic Field Calculations Including the Impact of Persistent Currents in Superconducting Filaments
}

\author{
M. Aleksa, S. Russenschuck, C. Völlinger \\ CERN, 1211 Geneva 23, Switzerland
}

\begin{abstract}
The magnetic field in the coils of superconducting magnets induces so-called persistent currents in the filaments. Persistent currents are bipolar screening currents that do not decay due to the lack of resistivity. The NbTi-filaments are type II superconductors and can be described by the critical state model [1]. This paper presents an analytical hysteresis model of the filament magnetization due to persistent currents which takes into account the changing magnetic induction inside the filament. This model is combined with numerical field computation methods, taking local saturation effects in the ferromagnetic yoke into consideration.
\end{abstract}

\section{Introduction}

The Large Hadron Collider (LHC) [2], a proton-proton superconducting accelerator, will consist of about $8400 \mathrm{su}-$ perconducting magnet units of different types, all operating in superfluid helium at a temperature of $1.9 \mathrm{~K}$. The applied magnetic field induces currents in the filaments that screen the external field. The filaments are made of type II hard superconducting material with the property that the magnetic field inside the filament does not vanish, but differs from the external field due to the screening effect of the persistent currents. Macroscopically, these persistent currents (that do not decay but persist due to the lack of resistivity) are the source of a magnetization $M(B)$ of the superconducting strands. One way to account for this magnetization is to mesh the coil in finite elements and solve the non-linear problem numerically by making use of a measured $M(B)$-curve. This approach has two main flaws: The numerical field computation has to be combined with a hysteresis model and the coil has to be discretized with highest accuracy also accounting for the existing gradient of the current density due to the trapezoidal shape of the cables, and the conductor alignment. Hence, we aimed for computational methods that avoid the meshing of the coil completely. In this paper, a model to calculate the magnetization of the superconducting strands is presented, taking into account alternating external fields, while keeping the magnetization vector parallel to the outside magnetic induction. Though the flux pinning is not described microscopically, this model fully considers the hysteretic behaviour of the superconducting filaments and predicts the measured magnetization. The method for the computation of persistent currents is based on the measured critical current density $j_{c}$ of the superconductors and calculates the filament magnetization by means of the critical state model [1]. It is combined with the coupled boundary element / finite element method (BEM-FEM-method) [3] which avoids the representation of the coil in the finite element mesh since the coil is positioned in the iron-free BEM domain. The fields arising from current sources in the coil are calculated by means of the Biot-Savart Law, while the surrounding non-linear iron yoke has to be meshed in finite elements. Hence, the discretization errors due to the finiteelement part in the BEM-FEM formulation are limited to the iron-magnetization arising from the yoke structure.

The input function $j_{c}(B, T)$ only depends on the material properties of the superconductor but is independent of geometrical parameters such as the filament diameter or shape, and the ratio $\lambda$ of the superconductor to total strand volume. The method reproduces the hysteretic behavior for arbitrary turning points in the magnet excitation cycle including minor loops. In order to account for the impact of the filament magnetization on the magnetic field an $M$-iteration as a function of $B$ is performed.

\section{The Magnetic Induction Inside the Filament}

The critical state model [1] postulates that the screening currents in the superconductor always flow with the critical current density $j_{c}$. Magnetization models for superconducting filaments can be found in literature (e.g. [4]). These models usually neglect the field-dependence of the critical current density inside the filaments and give expressions for the screening fields of slices of finite thickness. The model introduced in this paper includes changing current densities inside the filament and calculates the continuous course of the magnetic field by means of a differential approach. The dependence of the critical current density on the modulus of the magnetic induction $B=|\vec{B}|$ is given by the following fit [5], where $B_{c}=B_{c 0}\left(1-\left(T / T_{c 0}\right)^{1.7}\right)$ :

$$
j_{c}(B, T)=\frac{j_{c}^{\mathrm{ref}} C_{0} B^{\alpha-1}}{\left(B_{c}\right)^{\alpha}}\left(1-\frac{B}{B_{c}}\right)^{\beta}\left(1-\left(\frac{T}{T_{c 0}}\right)^{1.7}\right)^{\gamma} .
$$

The fit parameters for the LHC main-magnet cables are a critical current density at $4.2 \mathrm{~K}$ and $5 \mathrm{~T}$ of $j_{c}^{\text {ref }}=$ $3 \cdot 10^{9} \mathrm{~A} / \mathrm{m}^{2}$, an upper critical field $B_{c 0}=14.5 \mathrm{~T}$, a critical temperature of $T_{c 0}=9.2 \mathrm{~K}$, a normalization constant $C_{0}=27.04 \mathrm{~T}$ and the fit parameters $\alpha=0.57, \beta=0.9$ and 
$\gamma=2.32$

For small magnetic inductions $B$, where the persistent currents influence the field quality most, Eq. (1) strives for infinity with $B^{\alpha-1}=B^{-0.43} \sim 1 / \sqrt{B}$ for $B \rightarrow 0$. For the computation of the induction inside the filament, Eq. (1) was approximated around the actual value of the applied field $B_{\text {out }}=\left|\vec{B}_{\text {out }}\right|$ with the following function ( $T$ is constant):

$$
j_{c}(B, T) \sim j_{c}\left(B_{\text {out }}, T\right) \frac{\sqrt{B_{\text {out }}}}{\sqrt{B}} \equiv \frac{\mathcal{F}\left(B_{\text {out }}\right)}{\sqrt{B}} .
$$

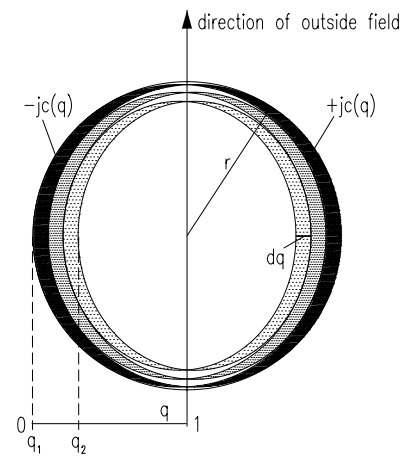

Fig. 1. Cross-section of one filament showing the intersecting ellipses of the critical current in the individual penetrations of one layer (here: virgin curve, non-fully penetrated state). For demonstration the slices are shown with finite thicknesses, while in the model $\mathrm{d} q \rightarrow 0$.

Be $q$ the relative penetration parameter which is zero at the surface of the filament and equals one in the center (see Fig. 1). From the expression of the perfectly uniform dipole field produced by two ellipses with opposite current densities shifted by the distance $r \mathrm{~d} q$ with respect to each other, the local field change $\mathrm{d} B(q)$ along the radial slice of thickness $r \mathrm{~d} q(\mathrm{~d} q \rightarrow 0)$ can be derived:

$$
\mathrm{d} B(q)=\xi \mu_{0} \mathcal{H} j_{c}(B(q)) \mathrm{d} q=\frac{\xi \mu_{0} \mathcal{H} \mathcal{F}\left(B_{\text {out }}\right) \mathrm{d} q}{\sqrt{B(q)}},
$$

where $r$ is the filament radius. The geometry factor $\mathcal{H}=$ $r / 2$ corresponds to the ideal screening field of two intersecting ellipses [4]. In our model $\mathcal{H}=r(2-2 \ln 2)=0.614 r$, correcting for the little spaces that are left when a round filament is filled with a series of intersecting ellipses (see Fig. $1,(\mathrm{~d} q \rightarrow 0))$. The parameter $\xi$ equals -1 in case of ramping up and $\xi=1$ for ramping down. In the first case, the orientation of the magnetic moment of the screening current is opposite to the orientation of the outside field $B_{\text {out }}$ and $B$ decreases inside the filament.

Equation (3) is a differential equation for $B(q)$, considering the dependence of $j_{c}$ on $B(q)$, that can be solved with the known boundary condition $B(0)=B_{\text {out }}$ in a closed analytical form, yielding:

$$
B(q)=\left(B_{\text {out }}^{3 / 2}+\frac{3}{2} \xi \mathcal{H} \mathcal{F}\left(B_{\text {out }}\right) \mu_{0} q\right)^{2 / 3}
$$

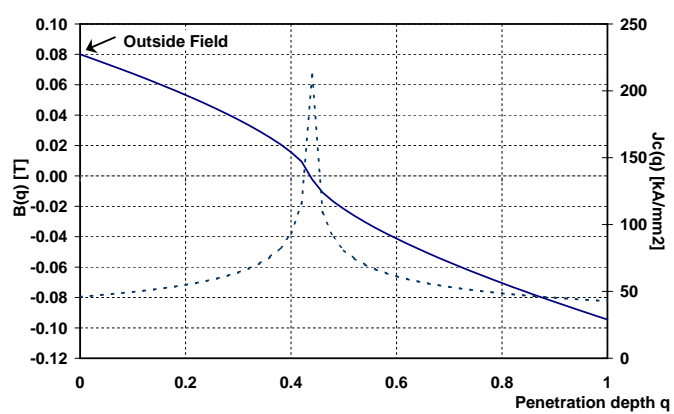

Fig. 2. Magnetic induction $B(q)$ as a function of the penetration depth $q$ (continuous line). The dashed line denotes the current density $j_{c}(q)$.

Figure 2 shows $B(q)$ according to Eq. (4) and the dependence of the critical current density $j_{c}(B(q))$ on the penetration depth $q$. The magnetic induction at $q=0$ equals the external field $B_{\text {out }}$. The shown field distribution is a fully penetrated state, reached for the case of increasing the external field from negative field values to $B_{\text {out }}=0.08 \mathrm{~T}$ $(\xi=-1)$. As is shown in Figure 2, this results in a decreasing field $B(q)$ along the penetration depth which produces an increase of $j_{c}(B(q))$ along $q$. At $B(q)=0$, the critical current density reaches its maximum value. There the strong increase of $j_{c}$ produces a sharp decline of $B(q)$. The course of $j_{c}(q)$ shows the importance of expressing $j_{c}$ as a function of $q$ rather than assuming a constant value.

\section{The Strand Magnetization and Hysteresis}

From the analytic expression for the magnetic induction $B(q)$ inside the filament, the magnetization due to the radial slice of current $j_{c}(q)$ between the penetrations $q_{i}$ and $q_{i+1}$ is derived. Individual slice magnetizations are needed to describe the hysteresis after changes of the ramp direction. Such a change $\left(\partial B_{\text {out }} / \partial t\right.$ changes sign $)$ will produce a new layer of screening currents with opposite polarity $(\xi$ switches sign). For small changes the new current layer will penetrate the filament only from $q_{1}=0$ to $q_{2} \leqslant 1$ while the currents inside persist. Figure 1 shows one such layer in the cross-section of a filament for the non-fully penetrated state. The values for $q_{i}$ are calculated using Eq. (4). For minor excitation loops, the magnetization is obtained as the superposition of $n$ different layers,

$$
M=\sum_{i=1}^{n} M_{i}=\sum_{i=1}^{n} \int_{q_{i}}^{q_{i+1}} m_{i}(q) \mathrm{d} q
$$

In Eq. (5) $M_{i}$ denotes the modulus of the magnetization $\vec{M}_{i}$; it has negative values if the orientation is opposite to the external field.

$M_{i}=\frac{4 r \xi}{\pi} \int_{q_{i}}^{q_{i+1}} j_{c}(B(q))(1-q)^{2} \mathrm{~d} q=\frac{4 r \xi \mathcal{F}}{\pi} \int_{q_{i}}^{q_{i+1}} \frac{(1-q)^{2}}{\sqrt{B(q)}} \mathrm{d} q$. 
Equation (6) can be solved analytically and - together with Eq. (4) - yields a closed expression for the filament magnetization:

$$
\begin{aligned}
& M_{i}=\frac{4 r B(q)}{5 \pi \mathcal{F}^{2} \mu_{0}^{2} \mathcal{H}^{3}}\left[B_{\text {out }}^{3}+\xi \mathcal{H} \mathcal{F} \mu_{0} .\right. \\
&\left.\cdot\left(\left(5-4 q+\frac{5}{4} q^{2}\right) \xi \mathcal{H} \mathcal{F} \mu_{0}-(q-4) B_{\text {out }}^{3 / 2}\right)\right]\left.\right|_{q=q_{i}} ^{q=q_{i+1}}
\end{aligned}
$$

Figure 3 presents computations of the filament magnetization according to Eq. (7) multiplied with the filling factor $\lambda$. The virgin curve and several hysteresis loops are displayed. The comparison of the calculation and the measured magnetization of a superconducting strand (dashed line) shows good agreement apart from the region of $B$ close to zero. There the difference between the magnetizations of one filament and a whole strand becomes significant. Since the outside field for each filament varies slightly due to the position in the strand cross-section and is additionally influenced by the field arising from the screening currents in the neighboring filaments, $B_{\text {out }}$ will be different for each filament according to its exact position. This results in a spread of filament magnetizations and hence in a smoothening of the region of $B$ close to zero. Since the Rutherford cables used in LHC magnets consist of many individual strands, this region will be smoothened out automatically due to the differences of $B_{\text {out }}$ at the individual strand positions within the coil.

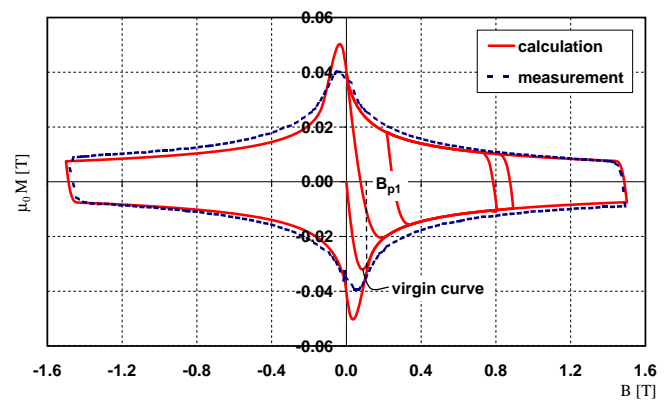

Fig. 3. Computed magnetization curve for one filament $(r=3.5 \mu \mathrm{m}$ $\lambda=1 / 2.95, T=1.9 \mathrm{~K}$ ) compared with measurements from a superconducting strand $[6]$.

\section{Virgin Curve and Fully Penetrated State}

From the expressions for the magnetic induction $B(q)$ (Eq. (4)) and the magnetization $M$ (Eqs. (6) and (5)) both parameters can be computed as a function of the penetration depth $q$. Figure 4 (upper plot), shows the values for increasing external fields $B_{\text {out }}(\xi=-1)$ for the virgin curve creating one layer extending from $q_{1}=0$ to $q_{2}\left(B_{\text {out }}\right)$. Depending on $B_{\text {out }}$, the field decreases until a certain penetration depth, where complete screening of the external field is obtained. The remaining part of the filament stays field free. In the lower plot the contribution $m(q)$ of a slice $\mathrm{d} q$ to the total magnetization $M$ is presented. The value of $M$ can be obtained by integrating the presented curves (see indication on the plot). Figure 5 illustrates the same quantities as in Fig. 4, but for a filament that has already experienced a negative outside field before (different history) and hence is fully penetrated. Since the currents inside the superconductor persist, there is a remaining negative field $B(q)$ inside, whereas in the case of the virgin curve the field remains zero for $q>q_{2}\left(B_{\text {out }}\right)$. The lower plot in Fig. 5 also explains why the maximum magnetization does not occur at $B_{\text {out }}=0$ : The magnetization is given by the integrated area $M$ under the $m(q)$ curve, which is biggest for small values of $B_{\text {out }} \neq 0$. This characteristic behaviour has already been observed in measurements (see Fig. 3), and is in good agreement with the calculations.

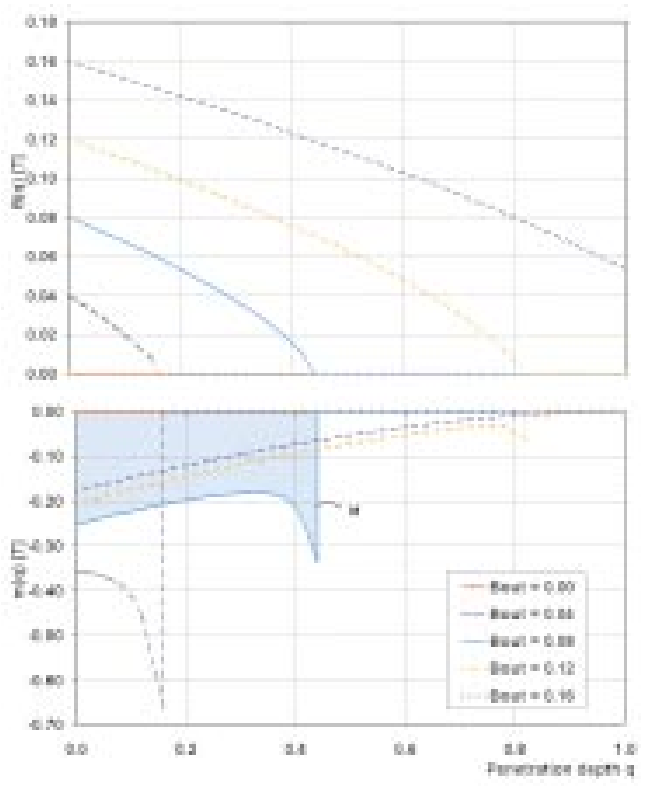

Fig. 4. Magnetic induction $B(q)$ and the magnetization contribution $m(q)$ as a function of the penetration depth $q$ for the virgin curve.

\section{Calculation of $B_{p 1}$}

The magnetic induction $B_{p 1}$ denotes the value of the outside magnetic induction where the modulus of the filament magnetization passes through its first maximum when ramping up on the virgin curve (see Fig. 3). Since the magnetization has been calculated in a closed analytical form (Eq. (7)), we can now derive the maximum of the virgin curve by solving $\partial M\left(B_{\text {out }}\right) / \partial B_{\text {out }}=0$. For the virgin curve the magnetization consists of only one layer, $n=1$, that penetrates from $q_{1}=0$ to $q_{2}=2 B_{\text {out }}^{3 / 2} /\left(\mathcal{H} \mathcal{F}\left(B_{\text {out }}\right) \mu_{0}\right)$, obtained by solving $B\left(q_{2}\right)=0$. In this region of $B_{\text {out }}=B_{p 1}$ we find that $\mathcal{F}^{\prime}\left(B_{\text {out }}\right) \cong 0$ and hence we obtain

$$
B_{p 1} \cong\left(\mathcal{H} \mathcal{F}\left(B_{p 1}\right) \mu_{0}\right)^{2 / 3} \frac{(15-5 \sqrt{5})^{1 / 3}}{2}
$$




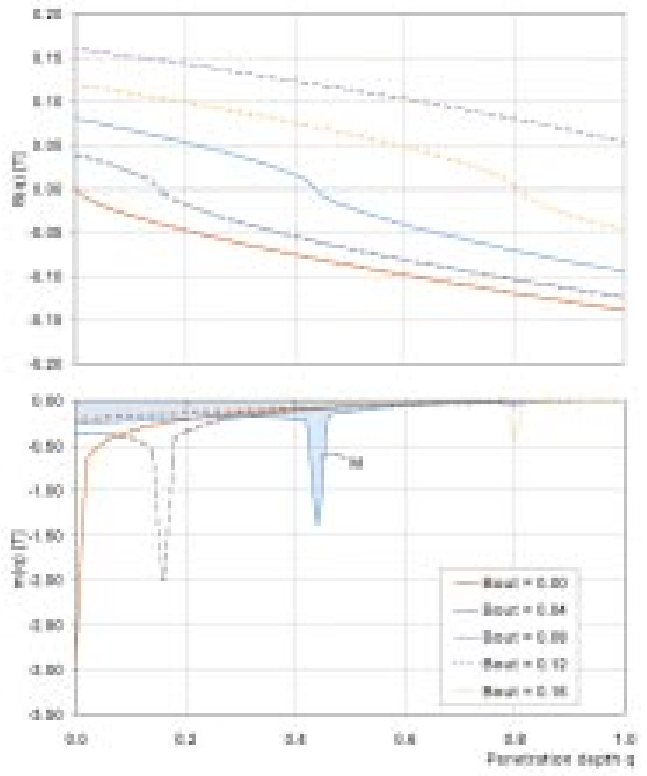

Fig. 5. Course of the magnetic induction $B(q)$ and the magnetization contribution $m(q)$ as a function of the penetration depth $q$ for a filament already been exposed to a magnetic field before (non-virgin curve)

$$
q_{2} \cong \sqrt{\frac{5}{6}-\frac{5 \sqrt{5}}{18}} \cong 0.46
$$

The recursive Eq. (8) yields a good estimate for $B_{p 1}$ after few iterations. From Eq. (9) can be seen that the maximum modulus of the magnetization does occur at a penetration depth of $q_{2} \cong 0.46$ rather than in the fully penetrated state. This fact is illustrated by the lower plot of Fig. 5, where the area will be maximal for $q \rightarrow 0.46$ (solid line). Note, that the value of $q_{2}$ is independent of the critical current fit, provided $\partial \mathcal{F}\left(B_{\text {out }}\right) / \partial B_{\text {out }} \cong 0$ (i.e. the critical current diverges with $1 / \sqrt{B_{\text {out }}}$, for $B_{\text {out }} \rightarrow 0$, see Eq. (2)).

\section{Combining the Model with Numerical Field Computation}

In the BEM-FEM coupling method the field in the nonmeshed air domain is the sum of the source fields from the superconducting coils ${ }^{1}$ and the reduced magnetic induction from the non-linear iron yoke. A third contribution, originating from the magnetization of the superconducting strands depending on the magnetic induction at the strand position has to be added to the source fields. Re-iterations are performed until convergence is obtained. For a description in detail see [7]. The presented model has been implemented into the ROXIE field computation program [3].

Figure 6 shows the results of a BEM-FEM calculation (obtained with ROXIE) of a quadrant of the LHC main quadrupole during ramping up $(I=730 \mathrm{~A})$. The magnetic induction was calculated as described above including the

${ }^{1}$ Calculated analytically by means of Biot-Savart's Law. influence of persistent currents. From this result, the multipoles in the aperture have been calculated. The global effect of the persistent currents in the strands on the field quality in the aperture of the magnet is a $\Delta b_{6}^{\text {pers }}=-3.8$ calculated at $17 \mathrm{~mm}$ reference radius and compares well to the measured values in the main quadrupole prototypes $\left(\left(\Delta b_{6}^{\text {pers }}\right)_{\text {meas }}=-3.5,[8]\right)$. For the definition of the field component $b_{6}$ see e.g. [2].

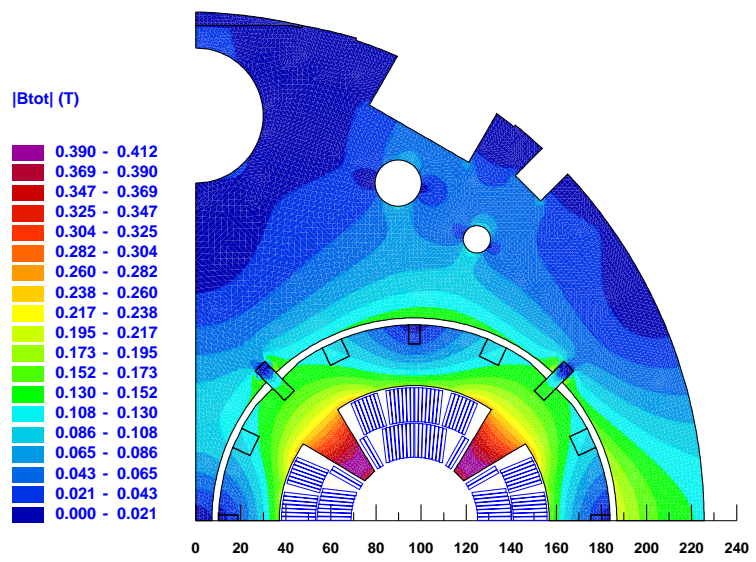

Fig. 6. Modulus of the magnetic induction $|B|$ of the main quadrupole including the stainless steel collar during ramping up $(I=730 \mathrm{~A})$. The effect of persistent currents has been taken into account.

\section{Conclusions}

A hysteresis model for the filament magnetization due to persistent currents has been developed and combined with the BEM-FEM [3] numerical field-computation method. Persistent currents influence the field quality of superconducting accelerator magnets. The model has been implemented into the ROXIE field computation program and is used to calculate the distortion of the magnetic field in the aperture of the superconducting magnets for LHC.

\section{References}

[1] C.P. Bean: Magnetization of High Field Superconductors, Review of Modern Physics, vol. 36, (1964).

[2] The LHC study group: The Yellow Book, LHC, The Large Hadron Collider - Conceptual Design, CERN/AC/95-5(LHC).

[3] S. Kurz and S. Russenschuck: The Application of the BEM-FEM Coupling Method for the Accurate Calculation of Fields in Superconducting Magnets, Electrical Engineering - Archiv für Elektrotechnik, vol. 82, no. 1, Berlin, Germany, (1999).

[4] M.N. Wilson: Superconducting Magnets, Monographs on Cryogenics, Oxford University Press, New York, (1983).

[5] L. Bottura: A Practical Fit for the Critical Surface of NbTi, 16th International Conference on Magnet Technology, (1999).

[6] S. Le Naour, et al.: Magnetization Measurements on LHC Superconducting Strands, ASC'98 App. Supercond. Conf., Palm Springs, USA (1998).

[7] C. Völlinger, M. Aleksa and S. Russenschuck: Calculation of Persistent Currents in Superconducting Magnets, Phys. Rev. ST Accel. Beams 3, 122402 (2000).

[8] J. Billan et al.: Performance of Series-Design Prototype Main Quadrupoles for the $\mathrm{LHC}$, Proc. $7^{\text {th }}$ Europ. Part. Accel. Conf., Vienna (2000). 